\title{
UM OLHAR PARA AS RELAÇŌES ENTRE AS MODELAGENS MATEMÁTICA E CIENTÍFICA A PARTIR DE UM ESTUDO BIBLIOGRÁFICO
}

\author{
Fabrício Fernando Halberstadt ${ }^{1}$ \\ Maria Cecilia Pereira Santarosa² \\ Isabel Krey Garcia ${ }^{3}$
}

\begin{abstract}
RESUMO
O presente trabalho debruça-se sobre como a relação entre as modelagens científica e matemática têm sido abordadas em trabalhos publicados em anais de eventos nas áreas de Educação Matemática e Educação em Ciências. Tem-se por pressuposto que a construção de modelos científicos geralmente requer algum nível de matematização. Descrevem-se os fundamentos, percursos e resultados de uma pesquisa de cunho qualitativo e do tipo bibliográfica, na qual se buscou investigar o seguinte problema de pesquisa: como são tratados os temas Modelagem Científica e Modelagem Matemática e suas relações em eventos nas áreas da Educação Matemática e Educação em Ciências? Foram analisados os anais das duas últimas edições do Encontro Paranaense de Modelagem Matemática - EPMEM, do último Encontro Paraense de Modelagem Matemática - EPAMM, da última Conferência Nacional sobre Modelagem na Educação Matemática - CNMEM e das duas mais recentes edições do Encontro Nacional de Pesquisa em Educação em Ciências ENPEC. Verifica-se que os trabalhos sobre modelagem na Educação Matemática analisados, de uma forma geral, não objetivam construir um modelo científico propriamente dito, buscam exclusivamente a construção de um modelo matemático. As modelagens descritas nos trabalhos publicados nas duas edições do ENPEC analisadas visam unicamente à construção dos modelos científicos. Esse resultado mostra a necessidade de pesquisas que possam caracterizar e diferenciar cada um dos processos, e buscar ações interdisciplinares que possam evidenciar as relações conceituais entre a matemática e as ciências, promovendo o avanço da aprendizagem em todos os níveis de ensino.
\end{abstract}

Palavras-chave: Modelagem Matemática. Modelagem Científica. Pesquisa Bibliográfica.

\footnotetext{
1 Doutorando do Programa de Pós-Graduação Educação em Ciências da Universidade Federal de Santa Maria. Professor do Campus São Vicente do Sul do Instituto Federal de Educação, Ciêcia e Tecnologia Farroupilha, São Vicente do Sul, RS, Brasil. Orcid iD: https://orcid.org/0000-0001-7685-6954. E-mail: ffhalberstadt@gmail.com

2 Doutora em Ensino de Física. Professora do Programa de Pós-Graduação Educação em Ciências da Universidade Federal de Santa Maria, Santa Maria, RS, Brasil. Orcid iD: http://orcid.org/0000-0002-7656-9100. E-mail: mcpsrosa@smail.ufsm.br

${ }_{3}$ Doutora em Ensino de Física. Professora do Programa de Pós-Graduação Educação em Ciências da Universidade Federal de Santa Maria, Santa Maria, RS, Brasil. Orcid iD: http://orcid.org/0000-0001-8080-6474. E-mail: ikrey69@gmail.com
}

Revista Exitus, Santarém/PA, Vol. 10, p. 01-30, e020067, 2020. 


\title{
AN EXAME OF THE RELATIONSHIP BETWEEN MATHEMATICAL AND SCIENTIFIC MODELING FROM A BIBLIOGRAPHICAL STUDY
}

\begin{abstract}
This paper focuses on how the relationship between scientific and mathematical modeling has been discussed in works published in proceedings of events in the areas of Mathematics Education and Science Education. Assuming that the construction of scientific models generally requires some level of mathematization, it describes the basis, the process and the results of a qualitative bibliographical study aimed at answering this question: How are the topics Scientific Modeling and Mathematical Modeling and their relations addressed in events in the areas of Mathematics Education and Science Education? The corpus of the investigation consisted of the proceedings of the following events: the last two editions of the Paranaense Meeting of Mathematical Modeling - EPMEM, the last Paraense Meeting of Mathematical Modeling - EPAMM, the last National Conference on Modeling in Mathematical Education - CNMEM and the two most recent editions of the National Research Meeting in Science Education - ENPEC. It appears that, in general, the papers on modeling in Mathematical Education analyzed do not aim at building a scientific model itself. Instead, they try to build exclusively a mathematical model. The modeling described in the papers published in the two editions of ENPEC are aimed only at building scientific models. These results show the need for research that can characterize and differentiate each one of these processes and seek interdisciplinary actions that can evince the conceptual relationships between mathematics and science, promoting the advance of learning at all levels of teaching.
\end{abstract}

Keywords: Mathematical Modeling. Scientific Modeling. Bibliographical research.

\section{UNA MIRADA A LA RELACIÓN ENTRE MODELOS MATEMÁTICOS Y CIENTÍFICOS DE UN ESTUDIO BIBLIOGRÁFICO}

\section{RESUMEN}

El presente artículo investiga sobre la relación entre los modelos científicos y matemática hay sido abordada en trabajos publicados en anales de eventos en las áreas de Educación Matemática y Educación en Ciencias. Hay la idea que la elaboración de modelos científicos generalmente requiere algún nivel de "matematização". Se describen los fundamentos, caminos y resultados de una investigación cualitativa y de carácter bibliográfica, en la cual se objetivo investigar el siguiente problema de investigación: ¿̇cómo son tratados los temas Modelaje Científica y Modelaje Matemática y sus relaciones en eventos en las áreas de la Educación Matemática y Educación en Ciencias? Fueron analizados los anales de las ultimas ediciones del Encuentro Paranaense de Modelaje Matemática - EPMEM, del último Encuentro Paraense de Modelaje Matemática - EPAMM, de la última Conferencia Nacional sobre Modelaje en la Educación Matemática-CNMEM y de las dos mas actuales ediciones del Encuentro Nacional de Investigación en Educación en Ciencias - ENPEC. Se verifica que los trabajos sobre modelaje en la Educación Matemática analizados, de una forma general, no objetivaban elaborar un modelo científico propiamente, buscan exclusivamente la construcción de un 
modelo matemático. Los modelajes descritos en los trabajos publicados en las dos ediciones del ENPEC analizadas objetivan únicamente a la elaboración de los modelos científicos. Ese resultado muestra la necesidad de investigaciones que permitan caracterizar y diferenciar cada uno de los procesos y buscar acciones interdisciplinares que puedan evidenciar las relaciones conceptuales entre la matemática y las ciencias, promoviendo el avanzo del aprendizaje en todos los niveles de enseñanza.

Palabras clave: Modelaje Matemática. Modelaje Científica. Investigación Bibliográfica.

\section{INTRODUÇÃO}

A busca por metodologias que potencializem o processo ensino e aprendizagem dos conceitos inerentes às áreas de Educação Matemática e Educação em Ciências nos diversos níveis e modalidades da educação brasileira não é recente. Várias pesquisas como Carvalho e Gil-Pérez (2011), Harres (1999) e Silva (2010) têm alertado para a presença nos espaços escolares e extraescolares de uma visão ingênua de ciência muito próxima ao senso comum: algo distante, de reponsabilidade de experts que detêm muito conhecimento, o qual é provado e, portanto, verdade inquestionável, imutável. Essa visão tem reflexo nas atividades desenvolvidas em sala de aula como, por exemplo, conforme lembram Fernandez et al. (2002), uma visão individualista da ciência pode conduzir a pouca valorização do trabalho coletivo. Além disso, conforme os mesmos autores, uma visão apenas disciplinar da ciência pode conduzir a um ensino compartimentalizado em que não há interação entre as disciplinas.

De forma semelhante às ciências, o ensino e aprendizagem de matemática tem constituído grande preocupação para pesquisadores, professores, alunos e a sociedade como um todo. Para D'Ambrosio (1996), decorre uma dificuldade em motivar os alunos para o estudo da matemática ao se considerar que esta é uma ciência esculpida há milhares de anos. Nesse sentido, esse autor afirma que, do ponto de vista da motivação contextualizada, a matemática ensinada nas escolas é morta, isto é, poderia ser tratada como um fato histórico.

Isto quer dizer que a pesquisa nessas duas áreas necessita ter atenção especial no que se refere às possibilidades metodológicas para o ensino e 
aprendizagem de ciências e matemática. Salienta-se que muitos esforços já têm sido realizados nas áreas de Educação Matemática e Educação em Ciências, entre os quais se podem citar as modelagens Científica e Matemática.

Nesse contexto, cabe indagar sobre a relação entre essas modelagens. Mais especificamente, o presente trabalho se debruça sobre o seguinte problema de pesquisa: como são tratados os temas Modelagem Científica e Modelagem Matemática e suas relações em eventos nas áreas da Educação Matemática e Educação em Ciências?

Tem-se como objetivo investigar se os trabalhos de modelagem matemática abordam conceitos científicos (e vice-versa) e de que formas isso acontece. Consiste de uma pesquisa qualitativa do tipo bibliográfica. A limitação por publicações em anais de eventos é devida pela forte presença de trabalhos do tipo relato de experiência nesse meio de divulgação, e que se pretendeu investigar no presente trabalho.

\section{MODELAGEM CIENTÍFICA E MATEMÁTICA}

Devido às características dessa pesquisa, ao longo do presente trabalho, busca-se analisar referenciais teóricos de duas principais linhas de trabalho em relação à modelagem. No que se refere à modelagem no ensino de ciências destacam-se, no presente trabalho, ideias de pesquisadores como John Gilbert, lleana María Greca e Marco Antonio Moreira. Quanto à modelagem matemática no ensino e aprendizagem de matemática, buscou-se ancorar o presente trabalho nas ideias de Rodney Carlos Bassanezi, Jonei Cerqueira Barbosa e Maria Sallet Biembengut.

Desde já, cabe esclarecer a visão adotada neste trabalho sobre a relação entre essas duas modelagens, a saber: a modelagem matemática está presente em muitos casos da construção de modelos e teorias científicas. Pensando na teoria de conjuntos poder-se-ia dizer que a modelagem matemática está contida na modelagem científica. 
FIGURA 1 - llustração sobre relação entre modelos matemáticos e científicos

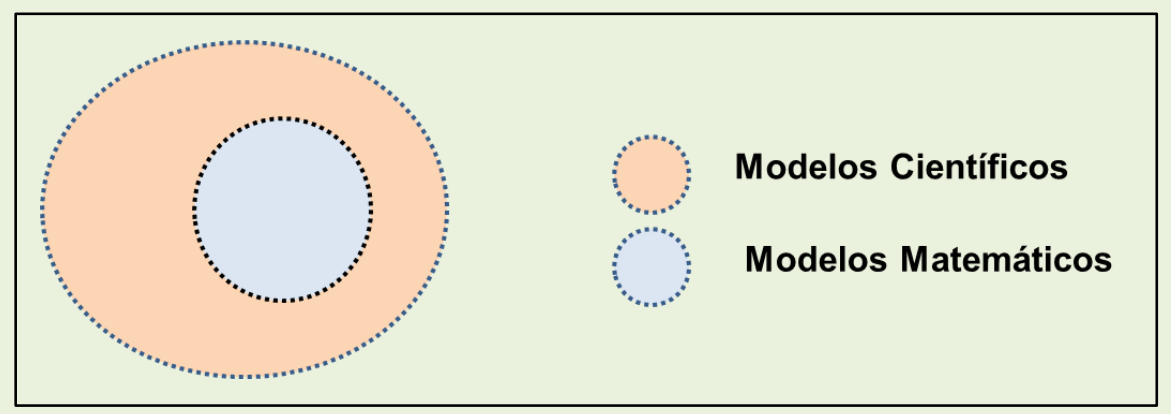

Fonte: os autores.

Por isso, a seguir, delineia-se pressupostos e resultados de cada uma dessas linhas de trabalho para, então, delinear suas convergências e retomar a posição assumida neste trabalho, ilustrada pela figura anterior.

\section{Modelagem Científica}

A perspectiva do trabalho com modelos científicos na área de Educação em Ciências vem se consolidando como uma oposição a visões positivistas outrora predominantes. Pensar que o trabalho científico produz modelos científicos que podem ser definidos como uma representação de uma ideia, um objeto, processo, evento ou sistema proporciona uma visão de ciência calcada no Construtivismo (GILBERT; BOULTER, 1998). Para esses mesmos autores, a modelagem científica consiste no processo de se obter esses modelos. Trata-se de pensar a ciência como construção humana, na qual o trabalho científico tem por objetivo produzir esses modelos pelos quais são ofertadas explicações a respeito do mundo que nos cerca. Tais modelos não são únicos, nem imutáveis, na verdade há uma comunidade intelectual que seleciona os melhores de acordo com o conhecimento produzido até aquele momento sendo, assim, passíveis de evolução (TOULMIN, 1977).

Moreira e Ostermann (1993) enfatizam que o conhecimento científico não é definitivo. O conhecimento que se tem hoje "está baseado em modelos e teorias inventados e que podem estar equivocados ou apenas parcialmente corretos" (MOREIRA; OSTERMANN, 1993, p. 115). 
Isso reforça a necessidade de se investigar possibilidades para a sala de aula de ciências, afinal, como já descrito anteriormente, devido à resistência à evolução, em muitos casos, há a prevalência do senso comum, nesse caso representado por práticas pautadas unicamente na transmissão do conhecimento científico. O que se quer dizer é que se o conhecimento científico não é imutável o ensino de Ciências também não deve ser.

Segundo Gilbert e Boulter (1998), o papel dos modelos e da modelagem merecem um enfoque próprio. Estes autores citam quatro motivos para justificar a adoção dessa posição. O primeiro refere-se à ubiquidade do termo modelo no campo da Educação em Ciências, afinal é empregado em diferentes momentos, para descrever distintas representações. Há incerteza sobre esse termo gerada pela variedade de modelos científicos, pela sua abrangência e validade. O segundo aspecto citado é o papel desempenhado pelos modelos na condução das ciências. Consequentemente, para uma educação em ciências mais próxima à realidade do trabalho científico é necessário enfrentar o tema dos modelos e da modelagem em sala de aula. Em terceiro lugar, apontam os autores, na perspectiva da psicologia cognitiva no campo da Educação em Ciências, há a referência da formação de modelos por indivíduo para a compreensão e aprendizagem das ciências, o que reforça a importância do estudo dos modelos e da modelagem nas ciências. Por último, os autores afirmam que os modelos definem um papel significativo na sala de aula, afinal proporcionam experiências e vivências diversas aos alunos que não observadas nos moldes tradicionais do ensino de Ciências.

Dessa forma, para Gilbert e Boulter (op. cit.), um modelo pode ser considerado como intermediário entre as abstrações da teoria e as ações concretas da experimentação. Um modelo permite, portanto, guiar o trabalho de investigação, selecionar e resumir dados, justificar resultados obtidos e viabilizar a sua comunicação.

Greca e Moreira (2000) afirmam que ao ensino de ciências deve ser incorporada a ideia de modelagem. Aprender as teorias científicas implica aprender como se faz a ciência e, portanto, é necessário "jogar o jogo". O 
que afirmam os autores é que o processo de modelagem pode contribuir para a aprendizagem da construção de modelos mentais 4 mais apropriados, coerentes com os modelos conceituais. Entretanto, enumeram que este ainda é um campo desconhecido e pouco trabalhado.

Dessa forma, pode-se concluir que a modelagem científica, em sala de aula, tem por objetivo principal possibilitar ao aluno que visualize 0 processo de construção da ciência. A aproximação da sala de aula com o trabalho científico pode favorecer o processo de ensino e aprendizagem à medida que o processo da modelagem dá sentido aos conceitos científicos e matemáticos.

\section{Modelagem Matemática}

Conforme lembra Bassanezi (2014), em suma, a modelagem matemática é a arte de expressar situações-problema do nosso meio por meio da linguagem matemática a fim de interpretar os fenômenos naturais e sociais. Esse processo consiste na criação de modelos. Na verdade, não só na matemática que se encontram modelos, mas também em outras áreas, tais como: Artes, História, Economia, Moda, Literatura, etc.

Para Biembengut e Hein (2011), no que tange à matemática, existem situações do mundo real que requerem soluções e decisões. Alguns desses problemas possuem solução quase que imediata, por meio da compreensão de uma matemática elementar como, por exemplo, a área de uma forma retangular ou o cálculo dos juros de uma determinada operação financeira. Todavia, há os problemas que necessitam de análise aprofundada, aparecem imersos em outras áreas, assim é necessário verificar as variáveis envolvidas como, por exemplo, os problemas tratados pela Biomatemática.

Seja qual for o caso, a resolução de um problema, em geral, quando quantificado, requer uma reformulação matemática detalhada. Nessa perspectiva, um conjunto de símbolos e relações matemáticas que procura traduzir, de alguma forma, um fenômeno em questão

\footnotetext{
${ }_{4}^{4}$ Referindo-se à Teoria dos Modelos Mentais de Johnson-Laird. Um modelo mental "é uma representação interna de informações que corresponde analogamente com aquilo que está sendo representado" (MOREIRA, 1996).
} 
ou problema de situação real denomina-se "modelo matemático" (BIEMBENGUT; HEIN, 2011, p. 12).

Dessa forma, Biembengut e Hein (op. cit.) afirmam que a modelagem matemática é o processo pelo qual se obtém o modelo matemático. Além de conhecimento matemático, esse trabalho demanda criatividade e intuição. Um modelo tende a ser mais sofisticado se maior for $o$ conhecimento matemático de quem o elabora.

Para Bassanezi (2014, p. 20), modelo matemático é um "conjunto de símbolos e relações matemáticas que representam de alguma forma $\mathrm{O}$ objeto estudado". Entretanto, o autor alerta que é necessário sempre ter em mente que os modelos obtidos são aproximações da realidade, e só assim a modelagem se torna eficiente. $O$ autor ainda alerta que a introdução exagerada de simbolismos matemáticos pode ser mais destrutiva que esclarecedora, ou seja, a utilização da modelagem matemática só é adequada se contribui para a compreensão do fenômeno abordado. Portanto, deve-se ter em mente quais são as hipóteses assumidas no processo de modelagem, as variáveis consideradas, bem como os possíveis erros cometidos devido às aproximações.

Para Bassanezi (op. cit.), a modelagem não se limita a um método científico de pesquisa, mas também pode consistir em uma estratégia de ensino e aprendizagem, a qual tem se mostrado muito eficaz. Entretanto, ainda é um campo a ser mais bem estudado e, sobretudo, levado à prática nas salas de aula.

Nesse sentido, o mesmo autor aponta alguns principais argumentos para a utilização da modelagem matemática no ensino e aprendizagem.

- Argumento formativo: à medida que enfatiza aplicações e a resolução de problemas contribui para desenvolver nos estudantes as capacidades de exploração e criação.

- Argumento de competência ética: a modelagem contribui para que na vida real os alunos tomem decisões, formando juízos próprios, por meio de aplicações com conceitos matemáticos. 
- Argumento de utilidade: a modelagem possibilita ao aluno utilizar ferramentas matemáticas para resolver problemas em diferentes situações e áreas.

- Argumento intrínseco: a modelagem, resolução de problemas e aplicações possibilitam ao aluno compreender nuances e facetas da matemática.

- Argumento da aprendizagem: os processos aplicativos da matemática garantem uma melhor compreensão de seus conceitos.

- Argumento de alternativa epistemológica: a modelagem matemática relaciona-se com a perspectiva da Etnomatemática proposta pelo educador Ubiratan D'Ambrósio, à medida que representa uma alternativa metodológica mais adequada às distintas realidades socioculturais.

Todavia, conforme Bassanezi (2014), também existem três tipos de obstáculos a serem superados no trabalho com modelagem, os quais são descritos a seguir.

- Obstáculos instrucionais: os cursos regulares costumam apresentar uma grade curricular fixa, na qual cada componente requer o estudo de conceitos específicos. Além disso, alguns professores parecem acreditar que - ensino da matemática deve tratar unicamente dos conceitos matemáticos, sem a necessidade de se verificar as suas relações com as demais áreas.

- Obstáculos para os estudantes: a modelagem matemática não é usual em sala de aula e, dessa maneira, as primeiras experiências nesse sentido podem representar dificuldades aos alunos, inclusive tornando-os apáticos para com essa metodologia. Trata-se de uma ruptura com o ensino tradicional caracterizado pela "transmissão de conhecimentos" do professor ao aluno. Além disso, o tema trabalhado na modelagem pode ser desinteressante a alguns alunos da classe.

- Obstáculos para os professores: Muitos professores não se sentem preparados para trabalhar com a modelagem matemática, por falta de conhecimento do processo ou medo de se confrontarem com situações em que Ihes possa faltar o conhecimento de como proceder. Além disso, 
possuem receio em "perder" muito tempo para preparar e desenvolver essas aulas e, assim, não cumprir o programa do curso.

Bassanezi (2014) lembra que, ao considerar a modelagem uma estratégia de ensino e aprendizagem, o mais importante dessa atividade é a descoberta das relações e conceitos matemáticos envolvidos e utilizados no processo, e não a descoberta do modelo em si. Entretanto, do ponto de vista do professor, poder-se-ia acrescentar que é necessário chamar a atenção para a capacidade do modelo matemático (mesmo que com margem de erro) predizer o que pode acontecer ou reexaminar o que aconteceu com o passar do tempo.

\section{O papel da Modelagem Matemática na Modelagem Científica}

Antes de tudo, cabe realizar uma retomada elencando os pontos convergentes entre as ideias apresentadas até o momento sobre modelagem científica e modelagem matemática. Uma primeira constatação está na convergência de suas definições e objetivos, isto é, na busca por uma representação da realidade observada (objetos, sistemas, etc.) que pode ser realizada por meio de conceitos científicos e ou matemáticos. Além disso, os modelos não são únicos, isto é, há uma diversidade de modelos que podem representar determinada situação.

Outro ponto convergente refere-se à defesa em torno de um ensino de ciências e matemática alicerçado em bases construtivistas. A modelagem científica busca contribuir para uma visão mais próxima do real trabalho científico, ou seja, uma ciência que não é acabada, "descoberta" por gênios, restrita a poucos, infalível. Por sua vez, a modelagem matemática também busca um aluno mais ativo em seu processo de aprendizagem e, além disso, construir conceitos matemáticos de forma dinâmica em um processo de cooperação entre o educador e o educando (KLÜBLER, 2010). De acordo com o mesmo autor, no caso da modelagem matemática também é almejada uma aproximação com o trabalho do matemático, pois se busca com ela construir conteúdos a partir dos 
problemas, e não o contrário como, geralmente, acontece nas salas de aulas.

Ambas as modelagens referem-se à necessidade da formação de professores para o trabalho em sala de aula, em especial a da Educação Básica brasileira. As modelagens são consideradas possibilidades metodológicas para o ensino. Entretanto, são raramente utilizadas pelos educadores, por motivos semelhantes, tais como: desconhecimento, angústia e receio em sua utilização (afinal, preveem instabilidade na construção dos conceitos), a formação inicial do professor em geral ainda é realizada de maneira disciplinar, tradicional e fragmentada.

Mas, afinal, por que abordar a relação entre as modelagens científica e matemática? Trata-se, sobretudo, da convicção da necessidade de trabalho interdisciplinar (ou minimamente contextualizado). A matemática não pode ser tratada de forma isolada, dando a entender que está simplesmente sujeita às necessidades das demais ciências. Pelo contrário, pode ser construída conjuntamente às demais ciências. Isso poderia parecer utópico, afinal, a realidade que se observa nas escolas é completamente diferente. Entretanto, acredita-se que a partir de uma melhor formação docente pode-se caminhar em direção a uma melhora nesse quadro.

Para Bassanezi (2014, p. 18), a ciência contemporânea é, todavia, resultado de "experiências planificadas e auxiliadas por teorias sujeitas à evolução". Isto quer dizer que a validade ou consistência de uma teoria, muitas vezes, depende diretamente da linguagem matemática que a envolve. O que se espera de um modelo matemático é que represente a realidade analisada a partir da idealização do fenômeno natural.

O mesmo autor ainda afirma que ciências como a Física, a Astrofísica e a Química já se encontram, atualmente, matematizadas de forma teórica. O processo de matematização das Ciências Biológicas encontra-se em expansão sendo utilizado para modelar a dinâmica de populações, a epidemiologia, a ecologia, a neurologia, a genética e os processos fisiológicos. Todavia, o mesmo ainda não é possível afirmar sobre as Ciências 
Sociais, apesar de já terem avançado significativamente na utilização de métodos estatísticos próprios.

Nesse sentido, Moreira (2014) converge com essa visão ao afirmar que a construção dos modelos científicos geralmente requer algum nível de modelagem matemática.

\begin{abstract}
A modelagem matemática está sempre presente na construção de teorias científicas, em particular de teorias físicas. Em decorrência, a modelagem matemática deveria também estar sempre presente no ensino de ciências, particularmente da Física (MOREIRA, 2014, p. 10).
\end{abstract}

Nesse sentido, Moreira (op. cit.) critica as práticas de ensino nas quais os modelos são apresentados nos seus formatos finais, cabendo aos alunos aceitarem essas construções científicas como se fossem definitivas e reproduzirem-nas. Em contraposição, defende que sejam abordadas, de forma conjunta, valorizando as relações existentes entre a matemática e as ciências. Poder-se-ia, portanto, dizer que se pretende que o aluno construa uma visão holística do conhecimento ou, de acordo com D'Ambrósio (1996), que permita ao aluno revisitar os caminhos da evolução da matemática a partir das necessidades sociais e históricas da humanidade.

Nesse sentido, uma discussão fundamental a ser realizada é o papel dos modelos matemáticos na educação científica. Para Barbosa (2009), diversas áreas como a Biologia, Química, Geografia, Geologia possuem modelos matemáticos. Entretanto, para o autor, é necessário discutir a visão de que o modelo matemático representa uma realidade do "mundo real", afinal pressupõe uma possível separação entre a matemática e a suposta realidade independente. Afirma que diferentes estudos têm se dedicado a explicitar que a matemática faz parte do mundo real e, por conseguinte, os modelos matemáticos são sempre uma representação enviesada.

Com isso, Barbosa (2009) sugere repensar a utilização dos modelos matemáticos na educação científica, afinal, segundo o autor, a concepção que aproxima a modelagem da realidade poderia potencializar determinadas visões no ensino e aprendizagem - mais especificamente, 
visões nas quais a matemática é a "rainha das ciências", isto é, sobrepõe-se a outros argumentos e valida o que é real ou não.

Dessa forma, por meio da análise de discursos pedagógicos, Barbosa (2009) define três ${ }^{5}$ diferentes utilizações (papéis) dos modelos matemáticos no ensino de ciências, a saber: como justificativa, como definição, como estruturante. O primeiro papel refere-se ao modelo matemático que é utilizado como justificativa para a introdução de um novo conceito. $O$ segundo caso - modelo matemático como definição - não subsidia a introdução de um conceito como no caso anterior, ele é o próprio conceito. Por fim, o terceiro caso se refere ao modelo matemático como estruturante, no qual há uma preocupação em clarificar as relações conceituais entre conceitos matemáticos e científicos e, assim, a matemática pode ter um discurso pedagógico mais ativo na educação científica. É Importante destacar que esse caso corrobora a visão de Pietrocola (2002) no que se refere à matemática como estruturante do conhecimento científico. Nesse caso, ela vai além de uma simples ferramenta para a ciência.

A relação entre as modelagens também pode ser observada no trabalho de Greca e Moreira (2001). Mais especificamente, os autores abordam as relações entre modelos físicos, matemáticos e mentais na perspectiva do ensino e aprendizagem de ciências. Ao debruçar-se sobre este trabalho, Santarosa (2013) afirma que se nota um papel subordinado da matemática no processo de entendimento dos fenômenos físicos.

Portanto, este papel de subordinação não exclui a Matemática do contexto da Física. Ao contrário, a relação de incorporação argumentada pelos autores caracteriza o Modelo Matemático como um conjunto que está contido num conjunto maior, referente ao conjunto dos Modelos Físicos, sendo que a intersecção destes conjuntos não é vazia, mas o próprio conjunto referente aos Modelos Matemáticos. No entanto, o complementar do conjunto referente aos Modelos Matemáticos com relação ao conjunto referente aos Modelos Físicos é a parte que não contém a Matemática, mas que está contida no universo Físico. O conjunto referente aos Modelos Mentais é ainda mais subordinante, por conter os demais (SANTAROSA, 2013, p. 44 - 45).

\footnotetext{
${ }^{5}$ Apesar de encontrar três tipos de utilizações, o autor defende que podem existir outras.
} 
Nesse sentido, Santarosa (2013) conclui que esta visão possibilita acreditar na integração dos conteúdos de Física e de Matemática. Acredita-se que, de forma análoga, também se pode pensar na relação entre a Matemática e a Biologia, a Matemática e a Química, e outras áreas do conhecimento. Isso significa que um problema físico, químico ou biológico, por exemplo, pode ser modelado por meio da matemática e este será um modelo científico.

A Figura 1 apresentada anteriormente ilustra essa concepção. O contorno pontilhado dos conjuntos representa a possibilidade de cada um deles ser expandida. Pietrocola (2002, p. 93) lembra que foi a partir do séc. XVII com Galileu, Kepler e outros, que "os fenômenos naturais começaram a ser sistematicamente expressos através de relações matemáticas". Cientistas, especialmente os físicos, desde então, têm se empenhado no processo que - autor chama de modelização matemática. Dessa forma, chama-se atenção para o emprego da modelagem matemática nas demais áreas científicas, trata-se de um processo em construção e em permanente evolução.

Portanto, em suma, busca-se, com a Figura 1, evidenciar que os modelos matemáticos são modelos científicos. Compreende-se que os modelos científicos geralmente requerem algum grau de matematização e, portanto, a modelagem envolve conceitos científicos e matemáticos, os quais estão intimamente imbricados. Dessa forma, assume-se, neste trabalho, que a compreensão de um modelo matemático passa pela compreensão das relações deste com os conceitos científicos envolvidos, e vice-versa quando se trata de um modelo científico que requer uma matematização.

Poder-se-ia, então, argumentar não ser necessário distinguir os termos modelagem científica e modelagem matemática de acordo com o que se pretende investigar no presente trabalho. Entretanto, acredita-se que essa distinção seja importante, pois na perspectiva teórica assumida, existem modelos científicos que não fazem uso da matemática, elementos do conjunto complementar ao conjunto dos Modelos Matemáticos, referidos por Santarosa (2013). Como exemplos pode-se citar o modelo teórico de 
calor, modelos atômicos, modelos de reação química e tipos de ligações, modelos desenvolvidos no âmbito da histologia, entre outros.

Dessa maneira, pode-se dizer que quando se pensa em modelagem matemática é necessário considerar também a modelagem científica. $O$ presente trabalho se debruça sobre essa relação por se acreditar em seu potencial para o ensino de ciências e matemática.

\section{METODOLOGIA}

O presente estudo possui viés qualitativo, pois sua principal característica é a interpretação dos dados coletados procurando escrutinálos exaustivamente de modo a compreendê-los considerando, também, as hipóteses geradas durante esse processo (MOREIRA, 2011). Conforme Gil (2002), a pesquisa descrita nesse trabalho pode ser classificada como bibliográfica à medida que é desenvolvida a partir de materiais já elaborados, mais especificamente artigos publicados em anais de eventos nas áreas de Educação Matemática e Educação em Ciências.

Pretende-se descrever uma investigação realizada em torno de como a modelagem científica é tratada nos trabalhos voltados à modelagem matemática e vice-versa. No que se refere à área de Educação Matemática, a partir do site Sociedade Brasileira de Educação Matemática (SBEM), pode-se identificar dois encontros regionais e uma conferência nacional, ambos específicos sobre a modelagem matemática, a saber: Encontro Paranaense de Modelagem Matemática - EPMEM (oito edições realizadas até o momento); Encontro Paraense de Modelagem Matemática - EPAMM (sete edições realizadas até o momento); Conferência Nacional sobre Modelagem na Educação Matemática - CNMEM (onze edições realizadas até o momento). Mais especificamente, definiu-se analisar os anais do VII EPMEM'6 e VIII EPMEM7', realizados nas cidades de Londrina/PR (em novembro de 2016) e Cascavel/PR (em outubro de 2018),

6 Os anais do VII EPMEM podem ser consultados em: https://drive.google.com/file/d/OB8BG_UHbVwUIUEl JZTF6YW5hekE/view

7 Os anais do VII EPMEM podem ser conferidos em: http://sbemparana.com.br/eventos/ index.php/EPMEM/VIIIEPMEM/schedConf/presentations 
respectivamente, o VII EPAMM8, realizado em Salinópolis/PA, em outubro de 2018 e o XI CNMEM ${ }^{9}$ ocorrido na cidade de Belo Horizonte/MG lem novembro de 2019).

Quanto à área de Educação em Ciências, não se identificou nenhum evento específico sobre modelagem científica. Dessa forma, a partir de consulta ao site da Associação Brasileira de Pesquisa em Educação em Ciências (ABRAPEC), optou-se por analisar os trabalhos do principal evento na área: o Encontro Nacional de Pesquisa em Ensino de Ciências - ENPEC (doze edições realizadas até o momento). Para tanto, serão analisados os trabalhos do XI ENPEC ${ }^{10}$ e do XII ENPEC ${ }^{11}$, realizados nas cidades de Florianópolis/SC (em julho de 2017) e Natal/RN (em junho de 2019), respectivamente.

\section{RESULTADOS}

O quadro a seguir apresenta o total de publicações encontradas nos anais dos eventos voltados à modelagem matemática.

QUADRO 1 - Publicações em anais de eventos de Educação Matemática

\begin{tabular}{|c|c|c|c|}
\hline Evento & Comunicações Científicas & Relatos de experiência & Total \\
\hline VII EPMEM & 31 & 35 & 66 \\
\hline VIII EPMEM $^{12}$ & 37 & 61 & 98 \\
\hline VII EPAMM & 9 & 12 & 21 \\
\hline XI CNMEM & 50 & 42 & 92 \\
\hline Total & 127 & 150 & 277 \\
\hline
\end{tabular}

Fonte: os autores.

8 Os anais do VII EPAMM podem ser consultados em: https://sites.google.com/view/viiepamm. Os anais do VI EPAMM (realizado em novembro de 2016) apresentam apenas pôsteres e, por isso, decidiu-se não analisar os trabalhos desta edição do evento.

9 Os anais do XI CNMEM podem ser conferidos em: http://eventos.sbem.com.br/index.php/cnmem/2019/schedConf/presentations

10 Os anais do XI ENPEC podem ser conferidos em: http://www.abrapecnet.org.br/enpec/xienpec/anais/index.htm

11 Os anais do XII ENPEC podem ser verificados em: http://abrapecnet.org.br/enpec/xiienpec/anais/busca_l.htm

12 O VIII EPMEM apresenta três modalidades de trabalhos, a saber: Relatos de experiência (46 trabalhos), Práticas de sala de aula (15 trabalhos) e Comunicações científicas (37). Como as categorias relato de experiência e práticas de sala de aula visaram o escrutínio de experiências realizadas em sala de aula, optou-se em unificá-las em uma só no presente quadro. 
A partir da leitura dos resumos dos trabalhos identificados incialmente nos anais desses eventos, selecionaram-se apenas os que tinham por objetivo discutir alguma proposta para a sala de aula que versasse sobre a modelagem, mesmo não tendo sido dinamizada. O quadro, a seguir, apresenta o quantitativo de propostas didáticas envolvendo modelagem matemática em cada um dos eventos.

QUADRO 2 - Propostas didáticas envolvendo Modelagem Matemática

\begin{tabular}{|c|c|c|c|}
\hline Evento & $\begin{array}{c}\text { Comunicações } \\
\text { Científicas }\end{array}$ & $\begin{array}{c}\text { Relatos de } \\
\text { experiência }\end{array}$ & Total \\
\hline VII EPMEM & 12 & 31 & 43 \\
\hline VIII EPMEM & 16 & 54 & 70 \\
\hline VII EPAMM & 3 & 6 & 9 \\
\hline XI CNMEM & 18 & 33 & 51 \\
\hline Total & 49 & 124 & 173 \\
\hline
\end{tabular}

Fonte: os autores.

Para uma análise inicial desses artigos, buscou-se responder a algumas questões comuns elaboradas pelos autores do presente projeto. Mais especificamente, perguntas prévias cujas respostas foram elaboradas a partir da leitura completa de cada artigo. Tais perguntas funcionaram como categorias a-priori de análise da pesquisa. São elas:

a) O que se pretende modelar?

b) Quais os principais conceitos utilizados na modelagem e inerentes a campos conceituais ${ }^{13}$ característicos da matemática?

c) Quais os principais conceitos utilizados na modelagem e inerentes a campos conceituais NÃO característicos da matemática?

d) É proposto algum tipo de trabalho conjunto com outras áreas do conhecimento? Com quem?

e) A quem é (ou foi) destinada essa proposta?

\footnotetext{
13 Referindo-nos à Teoria dos Campos Conceituais de Gérard Vergnaud, pois essa discussão é parte do trabalho de doutoramento do primeiro autor do presente trabalho, no qual se utiliza essa perspectiva teórica.
} 
O próximo passo foi a construção de planilhas ${ }^{14} \mathrm{com}$ as respostas para essas questões elaboradas a partir da leitura de cada trabalho.

Com isso, podem-se realizar algumas constatações iniciais:

- Há uma diversificação nos temas e problemas propostos nas atividades, o que evidencia a amplitude de possibilidades de trabalho com a modelagem em todos os níveis e modalidades de ensino.

- No que concerne aos conceitos matemáticos utilizados nas modelagens, pode-se verificar a presença recorrente de gráficos, equações e funções. Isso era esperado, afinal é característico da modelagem matemática buscar descrever um problema por meio de uma equação, o que pode ser feito a partir da constatação da correlação dos dados, muitas vezes, dispostos em uma tabela ou gráfico, e busca pela solução dessa equação. Embora, conforme lembram Burak e Aragão (2012), não exista modelo certo ou errado, o que os diferencia é o refinamento com que descrevem as situações estudadas. Assim, pode haver modelos que são maquetes, estruturas, desenhos, etc.

- Dos 173 trabalhos analisados, constatou-se que em 65 há uma preocupação em evidenciar conceitos inerentes a áreas distintas da matemática, cuja utilização se deu, em geral, com o intuito de contextualizar a modelagem matemática desenvolvida. Assim, apenas em 7 trabalhos há a participação de profissionais de outras áreas, os quais encontram-se resumidos no quadro a seguir.

14 Como os quadros de respostas a essas questões somam mais de trinta páginas, não é possível apresentá-los no presente trabalho. 
QUADRO 3 - Participação de profissionais em propostas de modelagem matemática

\begin{tabular}{|c|c|c|c|c|}
\hline Evento & Trabalho & $\begin{array}{l}\text { Profissional } \\
\text { participante (área } \\
\text { de formação) }\end{array}$ & $\begin{array}{l}\text { Conceitos utilizados } \\
\text { e inerentes a campos } \\
\text { conceituais NÃO } \\
\text { característicos } \\
\text { da matemática? }\end{array}$ & $\begin{array}{l}\text { Tipo de participação, atividades } \\
\text { desenvolvidas pelo profissional. }\end{array}$ \\
\hline \multirow{2}{*}{$\begin{array}{l}\text { VII } \\
\text { EPMEM }\end{array}$} & $\begin{array}{l}\text { (HUF, } \\
\text { BURAK, } \\
2016)\end{array}$ & $\begin{array}{l}\text { Profissional técnico } \\
\text { de uma estação } \\
\text { da Companhia de } \\
\text { Abastecimento do } \\
\text { Paraná. }\end{array}$ & Tratamento da água. & $\begin{array}{l}\text { Os alunos realizaram uma } \\
\text { visitação à Companhia de } \\
\text { Abastecimento do Paraná, no } \\
\text { qual foram atendidos por um } \\
\text { técnico da empresa, ao } \\
\text { qual solicitaram esclarecimentos } \\
\text { sobre suas dúvidas. }\end{array}$ \\
\hline & $\begin{array}{l}\text { (PISCHING, } \\
\text { KÖNIG, } \\
\text { BÖCKEL, } \\
2016 \text { ) }\end{array}$ & 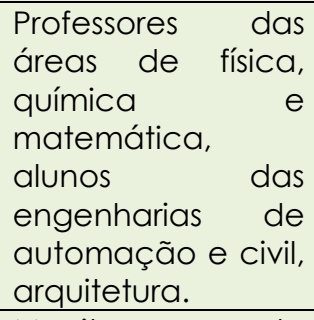 & Consumo, custo. & $\begin{array}{l}\text { Os autores esclarecem que o } \\
\text { planejamento da prática } \\
\text { realizado de } \\
\text { interdisciplinar com os referidos } \\
\text { profissionais. }\end{array}$ \\
\hline \multirow[t]{2}{*}{$\begin{array}{l}\text { VIII } \\
\text { EPMEM }\end{array}$} & $\begin{array}{l}\text { (MONZON, } \\
\text { SILVA, } \\
2018)\end{array}$ & $\begin{array}{lr}\text { Monitor } & \text { da } \\
\text { disciplina } & \text { cuja } \\
\text { modelagem } & \\
\text { relatada } & \text { foi } \\
\text { desenvolvida, o } & \\
\text { qual é licenciando } \\
\text { em Física e } \\
\text { primeiro autor do } \\
\text { trabalho. }\end{array}$ & $\begin{array}{l}\text { Movimento Circular } \\
\text { Uniforme, Movimento } \\
\text { Harmônico Simples. }\end{array}$ & Não detalhado no texto. \\
\hline & $\begin{array}{l}\text { (FERREIRA, } \\
\text { PEREIRA, } \\
\text { SOUSA, } \\
2018 \text { ) }\end{array}$ & $\begin{array}{l}\text { Colaboração de } \\
\text { alunos do curso de } \\
\text { Licenciatura em } \\
\text { Biologia. }\end{array}$ & Germinação. & $\begin{array}{l}\text { Ou autores referem que a } \\
\text { participação dos licenciandos } \\
\text { em Biologia foi fundamental } \\
\text { para o entendimento de termos } \\
\text { específicos dessa área. }\end{array}$ \\
\hline \multirow{3}{*}{$\begin{array}{l}\text { XI } \\
\text { CNMEM }\end{array}$} & $\begin{array}{l}\text { (BASTOS, } \\
\text { ROSA, } \\
2019 \text { ) }\end{array}$ & $\begin{array}{l}\text { Um professor de } \\
\text { Educação Física. }\end{array}$ & $\begin{array}{l}\text { Fichas de treino, } \\
\text { saúde física. }\end{array}$ & $\begin{array}{l}\text { Forneceu informações técnicas } \\
\text { sobre os processos que } \\
\text { deveriam ser considerados no } \\
\text { momento da prescrição de } \\
\text { treino físico. }\end{array}$ \\
\hline & $\begin{array}{l}\text { (CHAVES } \\
\text { et al., } \\
2019)\end{array}$ & $\begin{array}{l}\text { Participação } \\
\text { mergulhador } \\
\text { profissional. }\end{array}$ & $\begin{array}{l}\text { Fluxo laminar, } \\
\text { viscosidade, } \\
\text { conceitos inerentes a } \\
\text { mergulho. }\end{array}$ & $\begin{array}{l}\text { Realizou uma explicação } \\
\text { (consultoria) inicial sobre } \\
\text { mergulho equipado com } \\
\text { respiração por aparelhos, } \\
\text { abordando temas como } \\
\text { temperatura, turbidez, } \\
\text { viscosidade, vazão, relativos à } \\
\text { profundidade (portanto, } \\
\text { pressão) - no interior de } \\
\text { correntes marítimas, que } \\
\text { funcionam como dutos isolados } \\
\text { da massa oceânica. }\end{array}$ \\
\hline & $\begin{array}{l}\text { (BONOTTO, } \\
\text { SCHELLER, } \\
\text { 2019) }\end{array}$ & $\begin{array}{l}\text { Participação de } \\
\text { uma engenheira } \\
\text { florestal. }\end{array}$ & $\begin{array}{l}\text { Cubagem } \\
\text { madeira. }\end{array}$ & $\begin{array}{l}\text { Responsável por um seminário } \\
\text { sobre aspectos referentes à } \\
\text { biometria florestal. }\end{array}$ \\
\hline
\end{tabular}

Fonte: os autores. 
Nota-se que entre os sete trabalhos destacados, em quatro deles (HUF, BURAK, 2016), (BASTOS, ROSA, 2019), (CHAVES et al., 2019), (BONOTTO, SCHELLER, 2019) - a participação do profissional ocorreu sob a forma de uma consultoria inicial sobre os conceitos inerentes seja à sua área de formação seja à sua área de atuação. Apenas Pisching, König e BöckeL (2016) relatam que o planejamento da atividade proposta ocorreu de forma interdisciplinar, porém não detalham a participação de cada um desses profissionais. De forma semelhante, Ferreira, Pereira e Sousa (2018) mencionam que a participação de licenciandos em biologia foi essencial para a atividade, porém não adentram a como isso ocorreu e quais as implicações para a formação destes, bem como nos resultados obtidos. Em Monzon e Silva (2018) também não se tem especificado o trabalho do monitor (aluno de Licenciatura em Física) na atividade desenvolvida.

Ainda, buscou-se realizar uma categorização da natureza dos conceitos utilizados e inerentes a campos conceituais NÃO característicos da matemática. Com isso, procurou-se identificar as áreas do conhecimento presentes nas modelagens propostas nos trabalhos além da matemática. A seguir, apresentam-se as categorias delineadas, bem como os conceitos pertencentes a cada - entre parêntesis destacam-se as frequências de cada conceito, quando maiores que um.

- Física (total de 64 conceitos): aceleração, ambiente ideal, arrefecimento, caloria (3), corrente elétrica, densidade, elasticidade (2), empuxo (2), energia (2), energia elétrica (3), fluidos, fluxo laminar, força (4), gravidade (2), impulso, iodoterapia, lei de resfriamento de Newton, maré, massa (3), meia vida (3), movimento circular uniforme, movimento harmônico simples, movimento retilíneo uniformemente variado, pêndulo, ponto de orvalho, potência, queda livre, radiação, sistema massa-mola, temperatura (5), tempo (4), tensão, vazão, velocidade (6), velocidade média, viscosidade (2). - Biologia (total de 19 conceitos): biodiversidade, cacau, coleta seletiva, consumo da água, crescimento e desenvolvimento de plantas, cubagem de 
madeira, doação de órgãos, germinação, irrigação, jardim, lixo (3), reciclagem (3), recursos hídricos, sustentabilidade (2).

- Economia (total de 11 conceitos): câmbio, cesta básica, consumo, custo, exportação, importação, imposto, inflação (2), investimento financeiro, tributação de combustíveis.

- Saúde (total de 8 conceitos): alimentação saudável, caloria (2), fichas de treino, Índice de Massa Corporal - IMC, pirâmide alimentar, saúde, saúde física.

- Social (total de 4 conceitos): Funk, meios de transporte, mergulho, moda.

- Agropecuária (total de 3 conceitos): armazenamento de grãos em silos, processamento de café, produção de grãos.

- Tecnologia (total de 2 conceitos): pixel, programação.

- Geografia (total de 2 conceitos): densidade demográfica, precipitação pluviométrica.

- História (total de 2 conceitos): elementos de história ${ }^{15}$.

- Química (total de 1 conceito): tratamento da água.

- Outros (total de 2 conceitos): linguagem oral e escrita, cores primárias.

Cabe salientar que a categorização foi realizada de acordo com o tema abordado em cada artigo, afinal alguns conceitos ora foram agrupados em uma categoria ora em outra como, por exemplo, o conceito caloria pode ser abordado tanto no sentido nutricional (categoria saúde) bem como pode ser explorado seu conceito físico (categoria física) e poderia também na perspectiva da química.

A partir dessa categorização, é possível perceber uma predominância de conceitos da área da Física presentes nas modelagens matemáticas descritas nos artigos considerados. Acredita-se que essa aproximação se dê em virtude da natureza da área de Física, pois, conforme Pietrocola (2002), a Matemática é linguagem que permite ao cientista estruturar o seu pensamento. Cabe salientar que, para esse autor, a matemática não pode ser concebida como mera ferramenta das ciências. Além disso, destaca-se

15 Trabalho não especifica quais foram esses elementos históricos. 
a categoria relacionada à área de Biologia, cuja maioria dos conceitos refere-se ao campo da sustentabilidade. Acredita-se que este fato se dê em virtude da urgência e preocupação em discutir este tema.

Acredita-se ser possível e necessário, por meio da modelagem, ensinar e aprender conceitos inerentes a áreas distintas da matemática, em especial os das Ciências. O que se quer dizer é que os trabalhos sobre modelagem na Educação Matemática analisados, de uma forma geral, não objetivam construir um modelo científico. Na verdade, buscam exclusivamente a construção de um modelo matemático.

Dessa forma, considerando a diversidade de categorias encontradas, acredita-se que a busca pela interdisciplinaridade em trabalhos de modelagem matemática no ensino e aprendizagem seja um desafio a ser debatido nas áreas de Educação Matemática e Educação em Ciências. Não se trata de desmerecer os trabalhos publicados, pelo contrário, nestes percebe-se um empenho de seus proponentes de contextualizar os problemas abordados, inclusive no estudo de conceitos inerentes a áreas distintas de suas formações. Entretanto, com base nos resultados encontrados, verifica-se que há a necessidade de se empreender em discussões, atividades e estudos que abordem as modelagens que visam à resolução dos problemas (temas) propostos não apenas por meio de modelos matemáticos.

No que se refere às edições do ENPEC consideradas, a procura inicial pelos trabalhos foi realizada por meio da ferramenta de busca dos próprios sites os quais abrigam os anais desse evento. Nesse sentido, buscou-se por trabalhos que tivessem em seus títulos as palavras "modelo", "modelagem", "modelar", "modelado" ou "modelización". De forma análoga aos anais dos eventos da área de Educação Matemática analisados, inicialmente, foram lidos os resumos dos artigos encontrados em cada edição e selecionados os que tratavam de uma proposta com uso de modelagem para a sala de aula. Foram selecionados 20 trabalhos ( 13 do XI ENPEC e 7 do XII ENPEC) que versavam sobre propostas envolvendo modelagem científica. De forma análoga ao que foi feito com os trabalhos sobre modelagem matemática, 
as respostas (às perguntas realizadas) foram organizadas em um quadroresumo. Cabe salientar que as perguntas (a), (d) e (e) são idênticas às realizadas para os trabalhos sobre a modelagem matemática. As perguntas (b) e (c) foram adaptadas, a saber:

b- Quais os principais conceitos utilizados na modelagem e inerentes a campos conceituais característicos das ciências?

c- É utilizada modelagem matemática? São utilizados conceitos matemáticos na modelagem científica?

Com isso, a seguir, realizam-se algumas constatações a respeito destes trabalhos:

- Dois temas destacam-se no que se refere às propostas de modelagem: modelos celulares (5 trabalhos) e modelos sobre elementos químicos e - ou modelos atômicos (5 trabalhos). Estes modelos, costumeiramente, fazem parte do ensino de Biologia e Química, respectivamente, na Educação Básica. Entretanto, entende-se que essa concentração é um indício da necessidade da ampliação em torno da discussão, investigação e proposição de práticas envolvendo diferentes propostas de modelagem científica.

- Apenas dois artigos mencionam um trabalho conjunto com outros profissionais, Oliveira e Bonatto (2017) relatam a participação e auxílio técnico de um especialista (virologista) e Vicentini et al. (2017) descrevem ter contado com professores das áreas de Biologia, Física, Química e Matemática. Entretanto, não é explicitado neste último trabalho se foram abordados conceitos matemáticos no processo de modelagem ou qual foi a participação do professor da área de matemática. O quadro a seguir apresenta os trabalhos que abordaram conceitos matemáticos e - ou contaram com a participação de profissionais especialistas no tema abordado nas propostas desenvolvidas. 
QUADRO 4 - Participação de profissionais e ou conceitos científicos em propostas de modelagem científica

\begin{tabular}{|c|c|c|c|c|}
\hline Evento & Trabalho & $\begin{array}{l}\text { Profissional } \\
\text { participante } \\
\text { (área de } \\
\text { formação) }\end{array}$ & $\begin{array}{lr}\text { Conceitos utilizados e } \\
\text { inerentes a campos } \\
\text { conceituais } \\
\begin{array}{l}\text { característicos } \\
\text { ciências? }\end{array} \\
\text { das } \\
\end{array}$ & $\begin{array}{l}\text { Tipo de participação, } \\
\text { atividades } \\
\text { desenvolvidas pelo } \\
\text { profissional. }\end{array}$ \\
\hline \multirow{3}{*}{$\begin{array}{l}\text { XI } \\
\text { ENPEC }\end{array}$} & $\begin{array}{l}\text { (VICENTINI } \\
\text { et al., 2017) }\end{array}$ & $\begin{array}{l}\text { Professores de } \\
\text { Biologia, Física, } \\
\text { Química } \\
\text { Matemática. }\end{array}$ & Não informado. & $\begin{array}{l}\text { Consultor em uma } \\
\text { capacitação inicial da } \\
\text { equipe que compôs o } \\
\text { projeto discutido no } \\
\text { trabalho. }\end{array}$ \\
\hline & $\begin{array}{l}\text { (OLIVEIRA, } \\
\text { BONATTO, } \\
2017 \text { ) }\end{array}$ & $\begin{array}{l}\text { Auxílio técnico } \\
\text { de um virologista. }\end{array}$ & $\begin{array}{l}\text { Unidades de medida } \\
\text { de comprimento. }\end{array}$ & Não informado. \\
\hline & $\begin{array}{l}\text { (PEIXOTO et } \\
\text { al., 2017) }\end{array}$ & - & Proporção. & - \\
\hline $\begin{array}{l}\text { XII } \\
\text { ENPEC }\end{array}$ & $\begin{array}{l}\text { (SOUZA, } \\
\text { SALLES, } \\
\text { GAUCHE, } \\
2019)\end{array}$ & - & Grafo. & - \\
\hline
\end{tabular}

Fonte: os autores.

Nesse sentido, pode-se concluir, de forma equivalente ao já observado nos trabalhos sobre modelagem matemática, que, em geral, as modelagens descritas nos trabalhos publicados nas duas edições do ENPEC analisadas tratam prioritariamente conceitos científicos. Torna-se importante discutir possibilidades de interação entre as áreas científicas e a matemática, isto é, acredita-se ser imprescindível buscar compreender a relação existente entre essas duas modelagens. Para, então, avançar em propostas de ensino para a sala de aula que abordem o trabalho científico de forma não isolada da matemática, bem como propostas para o ensino de matemática que evidenciem a sua presença nas Ciências, isto é, propostas que evidenciem a matemática como estruturante do conhecimento científico (PIETROCOLA, 2002; BARBOSA, 2009).

A partir das constatações sobre os trabalhos publicados no VII EPMEM, VIII EPMEM, VII EPAMM, XI CNMEM, XI ENPEC E XII ENPEC, conclui-se que há um campo a ser estudado com mais ênfase: a modelagem matemática nas ciências. Pode-se dizer que a principal crítica que se faz a partir dessa análise reside em dois aspectos cuja motivação e consequências estão imbricadas, 
a saber: os trabalhos são, em sua grande maioria, iniciativas individuais de professores de uma mesma área (no caso da modelagem matemática professores de matemática e no caso da modelagem científica professores de ciências); com isso, um segundo ponto a destacar refere-se a não interação entre conceitos dessas duas áreas no processo de modelagem. Como dito anteriormente, mesmo nos trabalhos na área de modelagem matemática que mencionam alguns conceitos científicos, isso é feito com o intuito de contextualizar o trabalho desenvolvido, não havendo uma interação entre esses conceitos. Acredita-se ser importante refletir, realizar estudos e desenvolver práticas com o fito de relacionar a modelagem matemática nas ciências, promovendo um trabalho interdisciplinar entre a matemática e as ciências.

Novamente, não se trata de menosprezar os trabalhos analisados, afinal relatam resultados positivos na busca em potencializar o processo ensino e aprendizagem. De uma forma geral, os autores afirmam que os alunos envolvidos nas atividades demonstraram interesse pelas atividades, bem como construíram diferentes modelos para os problemas propostos, concluindo sempre a favor do uso da modelagem em sala de aula.

\section{CONSIDERAÇÕES FINAIS}

Ao longo do presente texto, buscou-se evidenciar a necessidade e importância de se discutir a relação entre as ciências e a matemática, mais especificamente no que se refere às modelagens científica e matemática. Compreende-se que a matemática não é uma simples ferramenta às demais ciências e, portanto, o ensino de matemática e ciências deve evidenciar o caráter estruturante da matemática nas demais ciências (PIETROCOLA, 2002; BARBOSA, 2009).

Descreveu-se uma pesquisa bibliográfica de cunho qualitativo a qual objetivou investigar as publicações em anais de eventos nas áreas de Educação Matemática e Educação em Ciências (VII EPMEM, VIII EPMEM, VII EPAMM, XI CNMEM, XI ENPEC e XII ENPEC). Inicialmente, localizaram-se os artigos publicados que tratavam sobre propostas para a sala de aula 
(efetivadas ou não). Para a categorização dos artigos encontrados, foram elaboradas perguntas para guiar a análise. Entre as quais duas foram determinantes para as conclusões encontradas no estudo: se os trabalhos envolvendo modelagem matemática utilizam conceitos científicos para a construção dos modelos decorrentes da modelagem (e vice-versa) e se houve a colaboração de áreas distintas no desenvolvimento da modelagem em sala de aula.

Verificou-se que os trabalhos que abordam a modelagem científica pouco exploram conceitos matemáticos. Os trabalhos considerados dos eventos na área de Educação Matemática (VII EPMEM, VIII EPMEM, VII EPAMM e XI CNMEM) objetivam a busca por uma modelagem matemática, isto é, buscam uma solução para o problema tratado na modelagem por meio do uso de conceitos matemáticos (utilizam-se especialmente de tabelas, gráficos e equações). Mesmo os que se preocupam em contextualizar o problema abordado por meio de conceitos característicos de outras áreas que não a matemática (65 de um total de 173 trabalhos) estão restritos ao trabalho dos professores que ensinam matemática. De forma análoga, dos trabalhos verificados nos eventos da área de Educação em Ciências (XI ENPEC e XII ENPEC) que objetivam a construção de modelos científicos, apenas $3 \mathrm{em}$ um total de 20 trabalhos exploram conceitos matemáticos. Não se está aqui, de forma nenhuma, buscando desvalorizar esses trabalhos. O presente trabalho é uma proposta complementar à investigação sobre este tema, a partir da discussão em torno de possibilidades de modelagem que avancem no sentido da compreensão das relações conceituais entre os conceitos matemáticos e científicos presentes no processo da modelagem.

Em suma, verificou-se que os trabalhos analisados tratam de experiências exitosas para $o$ ensino e aprendizagem de ciências e matemática. Entretanto, como não se debruçam sobre as relações conceituais entre essas duas áreas, concluiu-se que há uma temática a ser investigada. Uma possível explicação para esse cenário reside na falta de um trabalho conjunto com profissionais de diferentes áreas. Acredita-se que 
um possível encaminhamento seria a busca por uma modelagem interdisciplinar, caracterizada pelo trabalho conjunto de professores de diferentes áreas (no presente caso das áreas de ciências e matemática) visando à interação de conceitos científicos e matemáticos (JAPIASSU, 1976).

O estudo descrito nesse trabalho é um pontapé inicial, que objetivou apresentar uma visão geral sobre os trabalhos publicados nas áreas de Educação Científica e Educação Matemática. Um próximo passo que se pretende tomar refere-se a ampliar e atualizar o escopo de análise (artigos de periódicos e de anais de outros eventos). Outro encaminhamento possível se refere ao estudo da utilização da experimentação em trabalhos envolvendo modelagem matemática, com o intuito de verificar possíveis visões presentes sobre a ciência. Além disso, outra possível investigação futura consiste em buscar classificar trabalhos publicados sobre modelagem matemática, de acordo com o que propõe Barbosa (2009), classificando-se a utilização do modelo matemático como justificativa, definição ou estruturante da educação científica.

Por tudo isso, ao fim desse trabalho, cabe documentar os esforços registrados nos trabalhos analisados. Mesmo com cenários de pouca valorização profissional e de falta de infraestrutura das escolas brasileiras, veem-se professores e pesquisadores em busca de contribuir para a aprendizagem de matemática e ciências. Considera-se a modelagem uma possibilidade metodológica para a sala de aula de ciências e matemática que contribui para o desenvolvimento de um aluno mais ativo e empenhado cognitivamente com sua aprendizagem.

\section{REFERÊNCIAS}

BARBOSA, J. C. Modelagem e Modelos Matemáticos na Educação Científica. ALEXANDRIA Revista de Educação em Ciência e Tecnologia, v. 2, n. 2, p. 69-85, 2009.

BASTOS, T. A.; ROSA, M. Uma experiência de modelagem matemática no desenvolvimento de conceitos de análise combinatória. In: Conferência Nacional sobre Modelagem na Educação Matemática, 12., 2016, Belo Horizonte/MG. Anais... Belo Horizonte: Sociedade Brasileira de Educação Matemática, 2019. 
BASSANEZI, R. C. Ensino-aprendizagem com modelagem matemática. 4. ed. São Paulo: Contexto, 2014.

BIEMBENGUT, M. S.; HEIN, N. Modelagem matemática no ensino. 2. ed. São Paulo: Contexto, 2011.

BONOTTO, D. L.; SCHELLER, M. Cubagem de madeira com professores de matemática em serviço. In: Conferência Nacional sobre Modelagem na Educação Matemática, 12, 2016, Belo Horizonte/MG. Anais... Belo Horizonte: Sociedade Brasileira de Educação Matemática, 2019.

BURAK, D.; ARAGÃO, R. M. R. A modelagem matemática e relações com a aprendizagem significativa. Curitiba: CRV, 2012.

CARVALHO, A. M. P.; GIL-PÉREZ, D. Formação de professores de ciências: Tendências e inovações. 10. ed., São Paulo: Cortez, 2011.

CHAVES, R. et al. Ełapas da modelagem a partir da animação "Procurando Nemo". In: Conferência Nacional sobre Modelagem na Educação Matemática, 12, 2016, Belo Horizonte/MG. Anais... Belo Horizonte: Sociedade Brasileira de Educação Matemática, 2019.

D'AMBROSIO, U. Ensino-aprendizagem com modelagem matemática. São Paulo: Contexto, 1996.

FERNANDEZ, I. et al. Visiones deformadas de la ciência transmitidas por la enseñanza. Enseñanza de las Ciencias, v. 20, n. 3, p. 477-488, 2002.

FERREIRA, M. C.; PEREIRA, L. P. R.; SOUSA, B. N. P. A. Uma atividade de modelagem matemática para a análise da germinação de semente do pepino. In: Encontro Paranaense de Modelagem na educação Matemática, 8, 2018, Cascavel/PR. Anais... Cascavel: Universidade Estadual do Oeste do Paraná, 2018.

GIL, A. C. Como elaborar projetos de pesquisa. 4. ed. São Paulo: Atlas, 2002.

GILBERT, J. K.; BOULTER, C. J. Aprendendo ciências através de modelos e modelagem. In: COLINVAUX, D. (Org.) Modelos e Educação em

Ciências. Rio de Janeiro: Ravil, 1998.

GRECA, I. M.; MOREIRA, M. A. Mental models, conceptual models, and modelling. International Journal of Science Education, v. 22 n. 1, p. 1-11, 2000. 
GRECA, I. M.; MOREIRA, M. A. Mental, physical, and mathematical models in the teaching and leraning of physics. Science and Education, v. 86 n. 1, p. 106-121, 2001.

HARRES, J. B. S. Uma revisão nas pesquisas sobre as concepções de professores sobre a Natureza da Ciência e as implicações para o Ensino de Ciências. Investigações no Ensino de Ciências, v. 4, n. 3, 197 $211,1999$.

HUF, S. F.; BURAK, D. Modelagem matemática: reflexões sobre ações dos estudantes e do professor mediante a primeira experiência vivida. In: Encontro Paranaense de Modelagem na educação Matemática, 7, 2016, Londrina/PR. Anais... Londrina: Universidade Estadual de Londrina, 2016.

JAPIASSU, I. F. Interdisciplinaridade e patologia do saber. Rio de Janeiro: Imago, 1976.

KLÜBER, T. E. Modelagem matemática: revisitando aspectos que justificam a sua utilização no ensino. In: BRANDT, C. F.; BURAK, D.; KLÜBER, T. E. (Org.). Modelagem Matemática: uma perspectiva para a Educação Básica. Ponta Grossa: Editora UEPG, cap.5, p. 97-114, 2010.

MONZON, J. M. F.; SILVA, C. R. G. X. Modelagem de fenômenos físicos no ensino de trigonometria. In: Encontro Paranaense de Modelagem na educação Matemática, 8, 2018, Cascavel/PR. Anais... Cascavel: Universidade Estadual do Oeste do Paraná, 2018.

MOREIRA, M. A.; OSTERMANN, F. Sobre o ensino do método científico. Caderno Brasileiro de Ensino de Física. v.10, n. 2, p. 108-117, 1993.

MOREIRA, M. A. Modelos mentais. Investigações em Ensino de Ciências. v. 1, n. 3, p. 193-232, 1996.

MOREIRA, M. A. Pesquisa em ensino: aspectos metodológicos. In: Instituto de Física - UFRGS. Burgos: Universidade de Burgos, 2003. Disponível em: http://www.if.ufrgs.br/ moreira/pesquisaemensino.pdf>. Acesso em 24 jan. 2020.

MOREIRA, M. A. Metodologias de Pesquisa em Ensino. São Paulo: Livraria da Física, 2011.

MOREIRA, M. A. Modelos científicos, modelos mentais, modelagem computacional e matemática: aspectos epistemológicos e implicações para o ensino. Revista Brasileira de Ensino de Ciência e Tecnologia, vol. 7, n. 2, p. 1-20, 2014. 
OLIVEIRA, C. A. M.; BONATTO, M. P. O. Vírus e modelagem para a educação em saúde: uma investigação no Parque da Ciência/Museu da Vida/Fiocruz. In: Encontro Nacional de Pesquisa em Educação em Ciências, 11, 2017, Florianópolis/SC. Anais... Florianópolis: Universidade Estadual de Londrina, 2017.

PEIXOTO, B. G.; et al. Modelando a vida: pesquisa para a construção de oficina de modelagem de células no Parque da Ciência/Museu da Vida/ Fiocruz. In: Encontro Nacional de Pesquisa em Educação em Ciências, 11 , 2017, Florianópolis/SC. Anais... Florianópolis: Universidade Estadual de Londrina, 2017.

PIETROCOLA, M. A Matemática como estruturante do conhecimento físico.

Caderno Brasileiro de Ensino de Física. v. 19, n. 1, p. 93-114, 2002.

PISCHING, I.; KÖNIG, R. I.; BÖCKEL, W. J. Os Modelos Matemáticos emergentes a partir de Contas de Água. In: Encontro Paranaense de Modelagem em Educação Matemática, 7, 2016, Londrina/PR. Anais... Londrina: Universidade Estadual de Londrina, 2016.

SANTAROSA, M. C. P. Investigação da aprendizagem em Física Básica Universitária a partir de um ensino que integra situações e conceitos das disciplinas de Cálculo I e de Física I. 2013. 382f. Tese (Doutorado em Ensino de Física)-Universidade Federal do Rio Grande do Sul, Porto Alegre, 2013.

SILVA, B.V.C. A Natureza da Ciência pelos alunos do ensino médio: um estudo exploratório. Latin-American Journal of Physics Education, v. 4, n. 3, 2010.

SOUZA, P. V. T.; SALLES, P.; GAUCHE, R. O uso de modelos qualitativos como recurso didático para o Ensino de Ciências. In: Encontro Nacional de Pesquisa em Educação em Ciências, 12, 2019, Natal/RN. Anais... Natal: Universidade Federal do Rio Grande do Norte, 2019.

TOULMIN, S. La comprensión humana. I El uso colectivo y evolución de los conceptos. Madrid: Alianza Editorial, 1977.

VICENTINI, T.; et al. Museu de ciências e contextualização: um possível caminho para a alfabetização científica. In: Encontro Nacional de Pesquisa em Educação em Ciências, 11, 2017, Florianópolis/SC. Anais... Florianópolis: Universidade Estadual de Londrina, 2017.

Recebido em: 02 de março de 2019 Aprovado em: 20 de maio de 2020 